\title{
PDGFRB knockdown rescues the efficacy of chemotherapy in metastatic gastric cancer patients
}

\section{Type}

Research paper

\section{Keywords}

prognosis, neoadjuvant chemotherapy, PDGFRB, Metastatic gastric cancer

\begin{abstract}
Introduction

This study aimed to investigate the effect of PDGFRB on the efficacy of neoadjuvant chemotherapy (NAC) in metastatic gastric cancer (MGC).

\section{Material and methods}

The data from Gene Expression Omnibus (GEO) were analyzed to screen differentially expressed genes (DEGs). PDGFRB expression in cells were assessed by qRT-PCR and immunohistochemistry. Transwell, MTT and colony form assays were applied to determine the abilities of cell migration, cell invasion and cell proliferation. The association of core gene and PI3K/AKT pathway were identified through STRING database and western blot detection. Differences in overall survival (OS) among different patients were analyzed through Kaplan-Meier survival curve and prognostic factors were analyzed by Cox regression.
\end{abstract}

\section{Results}

High expression of PDGFRB and were found in gastric cancer through GEO data and MGC patients with combination chemotherapy resistance of docetaxel, cisplatin and S-1 (DCS therapy). Furthermore, the down-regulation of PDGFRB by PDGFRB shRNA or sunitinib malate could decrease cell migration, cell invasion and cell proliferation in resistant cells. Those findings were verified by in vivo experiments. Meanwhile, PDGFRB overexpression was accompany with the activation of PI3K/AKT pathway. In addition, the OS of patients in highly expressed PDGERB group were lower than that in lowly PDGFRB expressed group and PDGFRB, TNM staging and differentiation degree were the prognostic factors for MGC patients.

\section{Conclusions}

PDGFRB knockdown could rescue the efficacy of chemotherapy in MGC and PDGFRB could serve as a novel marker for the prognosis of MGC. 
PDGFRB knockdown rescues the efficacy of chemotherapy in metastatic gastric cancer patients

Running title: Role of PDGFRB in MGC

Yang Tian', Wei Song', Shuping Yang', Zheng Chen', Ming Li², Yan Zhang², Yanqing $\mathbf{L i}^{2^{*}}$

${ }^{1}$ Department of Oncology, Shandong Provincial Hospital Affiliated to Shandong University, Jinan 250000, Shandong, China

${ }^{2}$ Department of Gastroenterology, Shandong University, Qilu Hospital, Jinan 250000, Shandong, China

*Corresponding to: Yanqing Li, Department of Gastroenterology, Shandong University, Qilu Hospital, No. 107 West Wenhua Road, Jinan 250000, Shandong, China Email: kathleeni2@163.com

Tel.: +86 0531-68777153

Funding: This study was supported by Natural Science Foundation of Shandong Province, China (No. ZR2016HQ03(S.Y.)) and Natural Science Foundation of China (No. 81602332(S.Y.)). 


\section{Abstract}

Introduction: This study aimed to investigate the effect of PDGFRB on the efficacy of neoadjuvant chemotherapy (NAC) in metastatic gastric cancer (MGC).

Materials and methods: The data from Gene Expression Omnibus (GEO) were analyzed to screen differentially expressed genes (DEGs). PDGFRB expression in cells were assessed by qRT-PCR and immunohistochemistry. Transwell, MTT and colony form assays were applied to determine the abilities of cell migration, cell invasion and cell proliferation. The association of core gene and PI3K/AKT pathway were identified through STRING database and western blot detection. Differences in overall survival (OS) among different patients were analyzed through Kaplan-Meier survival curve and prognostic factors were analyzed by Cox regression.

Results: High expression of PDGFRB and were found in gastric cancer through GEO data and MGC patients with combination chemotherapy resistance of docetaxel, cisplatin and S-1 (DCS therapy). Furthermore, the down-regulation of PDGFRB by PDGFRB shRNA or sunitinib malate could decrease cell migration, cell invasion and cell proliferation in resistant cells. Those findings were verified by in vivo experiments. Meanwhile, PDGFRB overexpression was accompany with the activation of PI3K/AKT pathway. In addition, the OS of patients in highly expressed PDGERB group were lower than that in lowly PDGFRB expressed group and PDGFRB, TNM staging and differentiation degree were the prognostic factors for MGC patients.

Conclusions: PDGFRB knockdown could rescue the efficacy of chemotherapy in MGC 
and PDGFRB could serve as a novel marker for the prognosis of MGC.

Keywords: PDGFRB; Metastatic gastric cancer; Neoadjuvant chemotherapy; Prognosis 


\section{Introduction}

In 2016, gastric cancer (GC) was ranked as the second most common cause of cancer-related death and the fourth most common cancer in the world. About 1,400,000 people worldwide are diagnosed as GC each year, and approximately 850,000 people die from GC [1]. GC is a disease caused by multiple factors, including environmental and genetic factors, such as age, sex, smoking history, family history and Helicobacter pylori infection [2]. With the development of clinical research, the survival rate of GC patients has been significantly improved. For example, the five-year survival rate of patients was increased from $15 \%$ to $29 \%$ from 1975 to 2016 [3]. Surgery is the best choice for the treatment of GC [4], but a simple surgical is not suitable for the therapy of metastatic gastric cancer (MGC). For MGC, chemotherapy can effectively alleviate symptoms, improve the patients' life quality and increase the survival rate. Furthermore, multidrug chemotherapy precedes monotherapy in the survival rate, remission rate and symptom control $[5,6]$, but the five-year survival rate of metastatic gastric cancer is still only about $5 \%$. Seeking the markers for the diagnosis and prognosis of the diseases is significantly important [7]. It is necessary to find the biomarkers to predict the prognosis of MGC patients and regulate them for their further treatment.

Chemotherapeutic resistance refers to the resistance of tumor cells to chemotherapy drugs that often result in failure of chemotherapy. Due to the implementation of comprehensive diagnosis and treatment of tumor, chemotherapy resistance is an important cause of disease progression in patients. For example, patients with advanced 
ovarian cancer have a high level of primary resistance to first-line chemotherapy drug paclitaxel, and 50 percent of patients with a multidrug resistance have relapsed. Even though the combination of two or more chemotherapeutic drugs with different mechanisms is used to minimize the incidence of drug resistance and the increase of "cross resistance", side effects are still difficult to avoid. Chemotherapy resistance greatly limits the patient's clinical benefit. After a period of chemotherapy drug treatment, the tumor cells were adapted to change, such as epithelial cells to interstitial phenotype transformation, and cancer stem cell (CSC) amplification, etc., which caused secondary resistance. The more evidence that EMT and CSC activation are associated with the occurrence of drug resistance [8].

Platelet derived growth factor receptor (PDGFR) as a single transmembrane glycoprotein that belongs to the type III tyrosine protein kinase family, distributes in various kinds of cells, such as smooth muscle cells [9], fibroblasts [10], endothelial cells [11], glial cells and cartilage cells, etc. Moreover, PDGF and PDGFR are expressed in many human cancers, including colon [12], Head and neck squamous cell carcinoma (HNSCC) [13, 14], prostate [15], lung [16] and breast cancer [17]. PDGFRB gene is a kind of PDGFR, and plays an important role in promoting tumor cell proliferation, invasion, and new blood vessels form, which can result in metastasis of cancers. Considering that the platelet counts of patients suffering from advanced cancer are decreasing significantly, and PDGFR is closely related with platelet, PDGERB could function as a novel biomarker for MGC through regulating downstream pathways. 
Overall, we aimed to figure out the expression of PDGFRB in resistant cells of MGC, the influence on proliferation, migration and apoptosis of MGC cell lines and the reliability of PDGFRB as a prognostic marker.

\section{Materials and methods}

\section{Microarray data processing}

With "metastatic gastric cancer" and "chemotherapy" as keywords, "GSE31811" microarray data was screened as gene expression profiles for pre-analysis in the Gene Expression Omnibus (GEO) (http://www.nebi.nlm.nih.gov/geo). The platform was GPL6480: Agilent-014850 Whole Human Genome Microarray 4x44K G4112F (Probe Name version). Fresh-frozen samples of tumor tissue and background gastric mucosa tissue from 19 patients with gastric cancer by endoscopic biopsy before DCS therapy (chemotherapy with combination of docetaxel, cisplatin and S-1) were obtained. The expression data were used for subsequent analysis. Differentially expressed genes (DEGs) between cancer tissues and normal tissues were screened by $\mathrm{R}$ program language using GEO2R with $P<0.01$, |Fold change $\mid>2$.

\section{Study patients}

A total of $102 \mathrm{GC}$ patients who were enrolled at Shandong Provincial Hospital Affiliated to Shandong University during January 2013 to December 2017 were included in current study. Inclusion criteria included: (1) diagnosed through pathological examination before chemotherapy; (2) ECOG $\geq 2$, Karnofsky (KPS) $\geq 80$ points; (3) diagnosed with metastasis through clinical examination; (4) survival rate > 3 months; (5) signed informed consent. Exclusion criteria included: (1) incomplete clinical data; (2) with severe organ dysfunction; (3) in the phase of lactation or 
pregnancy.

\section{Therapeutic schedule}

MGC patients were treated with Neoadjuvant chemotherapy (NAC). Chemotherapy with combination of docetaxel, cisplatin and S-1 (DCS therapy) was performed for 2 courses. Each course lasted for 3 weeks. S-1 $\left(40 \mathrm{mg} / \mathrm{m}^{2}\right)$ was administered orally twice daily on days $1-14$, followed by a 7-day recovery period. Meanwhile, $60 \mathrm{mg} / \mathrm{m}^{2}$ of cisplatin and docetaxel mixture was given on day 8 of every course by 120 min of intravenous infusion. Patients were sorted into two groups: response and resistant group according to tumor regression rates (TRR) based on the Response Evaluation Criteria and Solid Tumors (RECIST) [18]. The patients in response group showed TRR > 10\% and the patients in resistant group showed TRR $<10 \%$ after 2 cycles of treatment.

\section{Cell culture, reagents and transfection}

After 2 cycles of treatment, patients in response and resistant group underwent biopsy from tumor. Tumor samples were treated with trypsin and then cultured in $90 \%$ RPMI-1640 with $10 \%$ FBS medium under condition of $37^{\circ} \mathrm{C}, 5 \% \mathrm{CO}_{2}$ for next cell experiments. Sunitinib malate was purchased from Bio Vision Inc. (San Francisco, CA, USA). Crenolanib was supplied by Arog Pharmaceuticals. Sorafenib tosylate was obtained from LC Laboratories (Woburn, MA). Drugs at a stock concentration of $10 \mu \mathrm{M}$ in dimethyl sulfoxide were dissolved in RPMI-1640 to the indicated final concentrations. Resistant cells were classified into three groups. The cells in DCS+sunitinib malate were treated with sunitinib malate and cells in vehicle group were treated with equivalent dimethyl sulfoxide. The cells in DCS group were without other treatment. The PDGFRB shRNA were synthesized (Thermo Fisher Scientific, Waltham, MA, USA) and cloned into lentiviral vector (LV3) and were then packaged with Lentivector 
Packaging Plasmid mix (pGag/Pol, pRev, and pVSV-G) to establish stable cell lines as previously described. Briefly, 293T Cells were cultured in a 6-well plate. The following day, cells were transfected with PDGFRB plasmids and Lentivector Packaging Plasmids with Lipofectamine 2000 Reagent (Life Technologies). After 48 h, the transfectants were selected with puromycin (Amresco, Cleveland, OH, USA) to gain the positive stably transfected clone.

\section{Quantitative real time polymerase chain reaction (qRT-PCR)}

Total RNA was extracted using the Trizol reagent (Thermo Fisher Scientific), and the obtained RNA was reverse-transcribed into cDNA using the GoScript reverse transcription system (\# A5001, Promega Corporation, WI, USA). Quantitative analysis of mRNA was performed using the QuantiTect SYBR Green PCR kit (Qiagen Company, Hilden, Germany) according to the manufacturer's instruction. The primer sequences of PDGFRB were as follows: forward 5'-GAACGCTCGCACGCCTAG-3' and reverse 5'-CATGGGAACACTGAGCTTCA-3'.

\section{Immunohistochemistry}

To detect the expression of PDGFRB, tissue sections from endoscopic biopsy were stained by LSABTM 2 Kit/HRP (DAKO, Tokyo, Japan). Briefly, after endogenous peroxidase and protein blocking reacts, the primary antibodies of PDGFRB (ab16868, Abcam, Cambridge, MA, US) were added, followed by HRP-labeled anti-rabbit IgG as the secondary antibody. And specimens were counterstained with hematoxylin.

\section{Transwell migration and invasion assay}

Transwell assay was performed for the ability of migration and invasion in the resistant cells. In short, the inserts were coated with $50 \mu \mathrm{L}$ Matrigel (BD Biosciences, Franklin Lakes, NJ, USA). Cells $\left(5 \times 10^{4}\right)$ were suspended in $100 \mu \mathrm{l}$ serum-free medium and then 
seeded on the upper floor of Transwell chambers (BD Biosciences). The lower chamber was added $500 \mu \mathrm{l}$ serum with $20 \% \mathrm{FBS}$. After $48 \mathrm{~h}$ of incubating at $37^{\circ} \mathrm{C}$ with $5 \% \mathrm{CO}_{2}$, the un-invaded cells were wiped with a cotton swab, and invaded cells were fixed in methanol and stained with $0.1 \%$ crystal violet. The number was counted under microscope. Each experiment was conducted in triplicate.

\section{MTT}

With $10 \%$ FBS of medium, the cells were prepared with a single cell suspension, and then they were inoculated into 96 orifice plates; each hole was filled with a volume of $200 \mu$ l. Constant temperature incubator with the condition of $37^{\circ} \mathrm{C}$ and $5 \% \mathrm{CO}_{2}$ were used to culture cells for 3 to 5 days. MTT solution $(5 \mathrm{mg} / \mathrm{ml}$ prepared with PBS, $\mathrm{pH}=$ 7.4) of $10 \mu \mathrm{l}$ was added into each hole to continue incubation for $4 \mathrm{~h}$, supernatant was absorbed in order to be removed from hole (suspended cells needed centrifugal suction first, then abandon supernatant). Each well plus $100 \mu \mathrm{DMSO}$ oscillated for $10 \mathrm{~min}$ so as to dissolve the crystal. $450 \mathrm{~nm}$ was chosen to detect the absorption value of various apertures on the enzyme-linked immunoassay, and the results were recorded. With time as the horizontal coordinate, the absorbance value was used to draw the growth curve of the cell.

\section{Colony formation assay}

Cells with specific treatment were collected and then placed onto a fresh 6-well plate. The cells were cultured for about 7 days to form colonies. Colonies were fixed by $100 \%$ methanol and stained by $0.1 \%$ crystal violet in $20 \%$ methanol for $15 \mathrm{~min}$. Colony-forming number was calculated under microscope.

\section{Western blot}

Cold phosphate buffer saline (PBS) was used to wash the cells twice, and the total 
protein was extracted with radio-immunoprecipitation assay (RIPA) lysis buffer. Concentration of the protein was then determined using the BCA kit (Thermo Fisher Scientific). Equal amounts of protein were re-dissolved in 10-14\% sodium dodecyl sulphate-polyacrylamide gel (SDS-PAG) and transferred to a closed nitrocellulose membrane (Amersham Biosciences, GE Healthcare, Milan, Italy). After that, the membrane was incubated with following antibodies: VEGFR (ab36844, 1: 1000, Abcam), PTEN (ab31392, 1: 1000, Abcam), PDGFRB (ab16868, 1: 2000, Abcam) and $\beta$-actin (ab8227, 1: 2000, Abcam) at $4^{\circ} \mathrm{C}$ overnight and then incubated with horseradish peroxidase-conjugated secondary antibody for $1 \mathrm{~h}$. The positive protein was detected using enhanced chemiluminescence reagent (Thermo Fisher Scientific) and analyzed by the ImageQuant LAS 4000 mini (GE Healthcare, Piscataway, NJ, USA).

\section{Tumor xenografts in nude mice}

Female 5/6-week-old BALB/c nude mice were bought from center of laboratory animal science of Guangdong. Treated cells with a concentration of $1 \times 10^{6} / \mathrm{ml}$ diluted in $0.1 \mathrm{ml}$ PBS were injected subcutaneously on the back flank of each mouse at day 0 . In the three groups, each group had 6 mice, and each one was injected with $1 \times 10^{6} / \mathrm{ml}$ cell. Tumor size was gauged with a caliper every 7 days. The tumor volume was computed via the formula (Volume=length $\times$ width $^{2} \times 0.5$ ). After 21 days observation, mice were executed, tumors were isolated to weigh by electro nic scales and perform immunohistochemistry assay and qRT-PCR assay. All experimental procedures were carried out on the basis of the ethical standards under a protocol approved by the Committee on Shandong Provincial Hospital Affiliated to Shandong University.

\section{Statistical analysis}

Measurement data were recorded in the form of average \pm standard deviation. 
Enumeration data were expressed as frequencies or percentages, and comparisons among multiple groups were made through Pearson chi-square test or Fisher's exact test when appropriate. The prognostic factors of MGC patients were screened using Cox regression analysis. Difference of the survival time between patients with different expression level of PDGFRB was tested by Log-rank method. All statistical analyses were done by $\mathrm{R} 3.4 .1$ program language software and $P<0.05$ signified statistical significance.

\section{Results}

\section{PDGFB expression was elevated in DCS therapy resistant cells}

The data from GSE31811 were analyzed using GEO2R to screen DEGs (differentially expressed genes) between cancer tissues and normal tissues. DEGs with $P<0.01$, |Fold change| $>2$ were selected, including 16 down-regulated genes and 5 up-regulated genes (Table 1). Compared with normal issues, PDGFB was highly expressed in cancer tissues. Besides, we detected the PDGFRB expression in tumor tissues from MGC patients and investigated the correlation between PDGFRB expression and clinic-pathological features. Results revealed that expression level of PDGFRB was related to TNM staging and differentiation degree, $P<0.05$ (Table 2).

Cells in response group and resistance group were transfected with empty vectors; resistant cells in shRNA group were transfected with PDGFRB shRNA. The expression of PDGFRB in resistant cells with DCS therapy was significantly higher than that in response group (Figure 1A). The resistant group had a higher immunohistochemical positive rate (Figure 1B). As showed in Figure 1C and 1D, we found that the invasion rate and migration rate of shRNA group was significantly lowest among three groups 
$(P<0.05)$, while the resistance group showed higher invasion and migration rate than response group $(P<0.05)$, which validated the up-regulated PDGFRB promoted cell migration and invasion in cancer. Subsequently, the influences of PDGFRB on the viability and proliferation of DCS therapy resistant cells were investigated. The outcomes of MTT assay indicated that DCS therapy resistance significantly promoted the proliferation of cells comparing with shRNA and response group (Figure 1E, $P<0.05)$. The colony number in resistant group were dramatically more than that in response group and approximately twice as much as shRNA group (Figure $1 \mathrm{~F}, P<0.05$ ).

\section{PDGFRB overexpression activated PI3K/AKT pathway}

A pre-computed database, STRING V9.0 was used to predict the MGC-related gene/protein-protein associations. Protein-protein interaction (PPI) network of MGC-related was gained from STRING. It indicated that PDGFRB was involved in the occurrence and development of MGC and PDGFRB played an important role in PI3K/AKT pathway (Figure 2A).

Western blot experiment indicated that the expressions of three different proteins, namely VEGFR, PTEN and PDGFRB in resistant group were all higher than those in response group. And resistant cells showed low level of VEGFR, PTEN and PDGFRB protein expressions after knock-downing PDGFRB (Figure $2 \mathrm{~B}, P<0.05$ ). In resistant cells, highly expressed PDGFRB was corresponding to the highly expressed VEGFR and PTEN and down-regulated PDGFRB was corresponding to the lowly expressed VEGFR and PTEN, which suggested that the AKT/PI3K pathway was activated.

Three kinds of inhibitors (sunitinib malate, creolanib, and sorafenib tosylate) were applied to treat resistant cells, we found that all of them could inhibit PDGFRB expression in different degrees compares with vehicle group (Figure $2 \mathrm{C}, P<0.05$ ). 
However, sunitinib malate showed the strongest effect among three inhibitors, namely sunitinib malate could effectively suppress the expression of PDGFRB. As mentioned before, the effect of DCS therapy would decrease during the early treatment of gastric cancer, and the resistance of DCS therapy would increase in a short period, DCS plus sunitinib malate group was required to increase the effect of DCS therapy. Figure 2D confirmed again that the expression of PDGFRB in DCS + sunitinib malate group was dramatically decreased compared with DCS or vehicle group $(P<0.05)$. It means that the supplement of sunitinib malate could increase the effect of DCS therapy and sensitize the drug susceptibility of cells.

PDGFRB inhibition could suppress the invasion, migration and proliferation of

\section{MGC cells}

The experimental results of Transwell showed that resistant cells treated with sunitinib malate (the group of DSC + sunitinib malate) showed decreased invasion and migration abilities compares with resistant cells in vehicle or DSC group $(P<0.05$, Figure 3A, B). Actually, it indicated that the inhibition of PDGFRB by adding sunitinib malate in resistant cells inhibited cell migration and invasion. In addition, MTT and colony formation assays were utilized to assess the cell viability and proliferation ability in three groups that prepared before. Results showed that cells viability in the DCS plus sunitinib malate group was lower than that in the DCS or vehicle group (Figure 3C, $P<0.05)$. Meanwhile, the colony numbers in DCS + sunitinib malate group were fewer than that in the DCS or vehicle group (Figure 3D, $P<0.05$ ). These findings indicated that the addition of sunitinib malate could enhance the influence of DCS in metastatic gastric cancer.

PDGFRB inhibition restrained tumor growth and predicted better prognosis 
To learn more about the effect of PDGFRB on cell function, in vivo experiments were performed. Resistant cells with different treatments were subcutaneously injected into the back flank of mice. Through continuous observation for 21 days, we found that the volume of tumor in DCS + sunitinib malate group was smaller compared with other two groups (Figure 4A, $P<0.05$ ). Similarly, the tumor weight in DCS + sunitinib malate group was lower than that in vehicle or DCS group (Figure 4B, $P<0.05$ ). Immunohistochemistry assay was carried out to detect the PDGFRB protein expression in tumor tissues of three groups' mice. Results showed that protein expression of the PDGFRB in DCS + sunitinib malate group was the lowest $(P<0.05$, when compared with vehicle or DCS group) (Figure 4C). Furthermore, qRT-PCR assay confirmed the results (Figure 4D, $P<0.05$ ).

Figure $4 \mathrm{E}$ and $4 \mathrm{~F}$ showed comparison of the survival rate between patients with high expressed PDGFRB and low expressed PDGFRB in two groups (with or without chemotherapy groups). As for the patients without chemotherapy, high expressed PDGFRB showed lower survival rate but the difference was not statistically significant $(P=0.25)$. As for the patients with chemotherapy, low expressed PDGFRB showed significantly higher survival rate $(P=0.00017)$. Additionally, COX regression analysis showed that the prognostic factors for MGC patients included TNM staging, differentiation degree and PDGFRB expression (Table 3). Above-mentioned results suggested that PDGFRB could be served as a prognostic indicator for patients with chemotherapy.

\section{Discussion}

Chemotherapy, especially the comprehensive treatment plays an important role in 
therapy of advanced cancer, including metastatic gastric cancer. There is a kind of membrane transporters, they bear the mass exchange between the inside and outside the cell membrane, substance metabolism, protect the normal physiological function of cells. In tumor cells, membrane pump function of the high expression of these proteins will reduce the antitumor drug "pump" out of cells or vesicles isolation, which results in the decrease of intracellular drug concentration or drug distribution changes leading to drug resistance of tumor cells. Gastric cancer resistance phenomenon has been a thorny problem in clinical treatment. At present, an increasing number of researches were aimed at settling drug resistance, including basing on microRNA [19, 20] or mRNA [21]. If gastric cancer drug resistance mechanism were thoroughly clarified, which will certainly promote the development of new drugs, increase the survival rate and improve the quality of life in patients with gastric cancer. As a result, the studies on basic and clinical resistance in gastric cancer are necessary. In our research, we aimed to figure out prognosis of metastatic gastric cancer and explore the effect of PDGFRB on chemotherapy in patients with metastatic gastric cancer.

PDGFRB is class III receptor tyrosine kinases (RTK) functioning in the development of normal connective tissue cells. In non-small cell lung cancer (NSCLC), alterations in PDGFR are markers of poor prognosis and efficient targets of these receptors are yet to be achieved [22]. Aberrant activation of PDGFR signaling due to gene amplification, translocation and activating mutation, has been found in various solid tumors [23]. PDGFR A and B often form heterodimers to activate downstream signaling [23]. The 
expression of PDGFRB mRNA was higher in colorectal adenoma than that in normal tissues, suggesting that PDGFRB has a role in the diagnosis of rectal adenocarcinoma [24]. When the cervical intraepithelial neoplasia develops to the micro-invasion cancer, PDGFRB protein expression is increased, which is beneficial to the pathological diagnosis and prognosis evaluation [25]. In addition, PDGFR in the tumor stroma have attracted attention as interesting drug target due to their function as regulators of tumor transvascular transport, tumor interstitial fluid pressure and tumor drug uptake [26]. PDGFRB expression induced by inflammatory cytokines could promote growth of intestinal smooth muscle cells and control of PDGFRB expression might be beneficial in chronic intestinal inflammation [9]. We found PDGFB expression was elevated in DCS therapy resistant cells. This result was similar to the study of Kodama M et al. that PDGFRB expression was associated with lymphatic metastasis in gastric carcinoma [11]. Combing with our research results, PDGFRB could be considered as a new biomarker in MGC prognosis.

Various signaling pathways were involved in progress of cancer. Jedroszka D et al. reported that the expression of Notch pathway members could be considered as useful biomarkers for predicting prognosis in renal carcinoma and specific member of the Notch pathway was related to the specific subtype of renal carcinoma [27]. In current study, we also found that PDGFB overexpression activated PI3K/AKT pathway and PDGFRB inhibition could suppress the invasion and migration of MGC cells. Consistent to our findings, Wang $\mathrm{H}$ et al. reported the over-expression of the PDGFRB 
in endothelial progenitor cells (EPCs) enhanced the PDGF-BB-induced proliferation, migration and angiogenesis of EPCs though the PI3K/AKT signaling pathway. Moreover, the PI3K/AKT signaling pathway was required for PDGFRB over-expression to enhance these PDGF-BB-induced phenotypes [28]. Similarly, PDGFB inhibited apoptosis in the pulmonary arterial endothelial cells by stimulating the phosphorylation of both AKT and STAT3, and the PI3K/AKT signaling pathway served as an up-stream participant in the STAT3 activation stimulated by PDGFB [29].

Though PDGFRB was expressed in various cancers, this study was firstly revealed PDGFRB was highly expressed in DCS therapy resistant cells compared to in DCS therapy response cells. Furthermore, follow-up study of MGC patients confirmed that for MGC patients with chemotherapy, lowly expressed PDGFRB indicated higher survival rate compared with highly expressed PDGFRB. Obviously, more and more genes associated with prognosis were discovered [30]. For example, Feng W et al. found high expression of lncRNA RMRP was related with a relatively poor prognosis in glioma patients [31]. Certainly, there are still several limitations in this study. Firstly, we could further study the influence of PDGFRB on chemotherapy resistance of MGC cells directly by knock-downing PDGFRB replacing PDGFRB inhibitor. Secondly, the concrete functional mechanisms between PDGFRB and the PI3K/AKT pathway need to further be investigated.

In conclusion, PDGFRB expression was elevated in DCS therapy resistant cells, which can active the PI3K/AKT signaling pathway. PDGFB inhibition suppressed metastasis 
and growth of resistant cells. PDGFRB, as a novel molecular marker, can predict the prognosis of metastatic gastric cancer patients treated with neoadjuvant chemotherapy.

\section{Conclusion}

A new gene PDGFRB in MGC is explored in current study. This study verifies PDGFRB knockdown can rescue the efficacy of chemotherapy in MGC and finds low expression of PDGFRB is corresponding to a higher survival rate, indicating PDGFRB can serve as a novel marker for the prognosis of MGC.

\section{Acknowledgements}

We would like to give our sincere appreciation to the reviewers for their helpful comments on this article.

\section{Conflict of interest}

The authors have declared that no competing interests exist. 


\section{References}

1. Arnold M, Laversanne M, Brown LM, Devesa SS, Bray F. Predicting the Future Burden of Esophageal Cancer by Histological Subtype: International Trends in Incidence up to 2030. Am J Gastroenterol 2017;112:1247-1255.

2. Karimi P, Islami F, Anandasabapathy S, Freedman ND, Kamangar F. Gastric cancer: descriptive epidemiology, risk factors, screening, and prevention. Cancer Epidemiol Biomarkers Prev 2014;23:700-713.

3. Siegel R, Ma J, Zou Z, Jemal A. Cancer statistics, 2014. CA Cancer J Clin 2014;64:9-29.

4. Blakely AM, Miner TJ. Surgical considerations in the treatment of gastric cancer. Gastroenterol Clin North Am 2013;42:337-357.

5. Pasini F, Fraccon AP, De MG. The role of chemotherapy in metastatic gastric cancer. Anticancer Research 2011;31:3543-3554.

6. Hacker U, Lordick F. Current standards in the treatment of gastric cancer. Dtsch Med Wochenschr 2015;140:1202-1205.

7. Sayan S, Kotan D. Levels of brain natriuretic peptide as a marker for the diagnosis and prognosis of acute ischemic stroke. Arch Med Sci Atheroscler Dis 2016;1:e16-e22.

8. Shibue T, Weinberg RA. EMT, CSCs, and drug resistance: the mechanistic link and clinical implications. Nat Rev Clin Oncol 2017;14:611-629.

9. Nair DG, Miller KG, Lourenssen SR, Blennerhassett MG. Inflammatory cytokines 
promote growth of intestinal smooth muscle cells by induced expression of PDGF-Rbeta. J Cell Mol Med 2014;18:444-454.

10. Sumida T, Kitadai Y, Shinagawa K, et al. Anti-stromal therapy with imatinib inhibits growth and metastasis of gastric carcinoma in an orthotopic nude mouse model. Int J Cancer 2011;128:2050-2062.

11. Kodama M, Kitadai Y, Sumida T, et al. Expression of platelet-derived growth factor (PDGF)-B and PDGF-receptor beta is associated with lymphatic metastasis in human gastric carcinoma. Cancer Sci 2010;101:1984-1989.

12. Yuge R, Kitadai Y, Shinagawa K, et al. mTOR and PDGF pathway blockade inhibits liver metastasis of colorectal cancer by modulating the tumor microenvironment. Am J Pathol 2015;185:399-408.

13. Schultz JD, Muhlheim K, Erben P, et al. Chemotherapeutic alteration of VEGF-/PDGF- and PDGF-Ralpha/beta expression by imatinib in HPV-transformed squamous cell carcinoma compared to HPV-negative HNSCC in vitro. Oncol Rep 2011;26:1099-1109.

14. Schultz JD, Rotunno S, Riedel F, et al. Synergistic effects of imatinib and carboplatin on VEGF, PDGF and PDGF-Ralpha/ss expression in squamous cell carcinoma of the head and neck in vitro. Int J Oncol 2011;38:1001-1012.

15. Uehara H, Kim SJ, Karashima T, et al. Effects of blocking platelet-derived growth factor-receptor signaling in a mouse model of experimental prostate cancer bone metastases. J Natl Cancer Inst 2003;95:458-470. 
16. Antoniades HN, Galanopoulos T, Neville-Golden J , O'Hara CJ. Malignant epithelial cells in primary human lung carcinomas coexpress in vivo platelet-derived growth factor (PDGF) and PDGF receptor mRNAs and their protein products. Proc Natl Acad Sci U S A 1992;89:3942-3946.

17. Yi B, Williams PJ, Niewolna M, Wang Y, Yoneda T. Tumor-derived platelet-derived growth factor-BB plays a critical role in osteosclerotic bone metastasis in an animal model of human breast cancer. Cancer Res 2002;62:917-923.

18. Eisenhauer EA, Therasse P, Bogaerts J, et al. New response evaluation criteria in solid tumours: revised RECIST guideline (version 1.1). Eur J Cancer 2009;45:228-247.

19. Dehghanzadeh R, Jadidi-Niaragh F, Gharibi T , Yousefi M. MicroRNA-induced drug resistance in gastric cancer. Biomed Pharmacother 2015;74:191-199.

20. Shang Y, Zhang Z, Liu Z, et al. miR-508-5p regulates multidrug resistance of gastric cancer by targeting ABCB1 and ZNRD1. Oncogene 2014;33:3267-3276.

21. Li X, Yao R, Yue L, et al. FOXM1 mediates resistance to docetaxel in gastric cancer via up-regulating Stathmin. J Cell Mol Med 2014;18:811-823.

22. Naidu S, Shi L, Magee , et al. PDGFR-modulated miR-23b cluster and miR-125a-5p suppress lung tumorigenesis by targeting multiple components of KRAS and NF-kB pathways. Sci Rep 2017;7:15441.

23. Chen PH, Chen X, He X. Platelet-derived growth factors and their receptors: 
structural and functional perspectives. Biochim Biophys Acta $2013 ; 1834: 2176-2186$.

24. Osama A, Sabry D, Hassany SM, Abdelmoneim SS, Sabry A. SIRT-1expression is associated with expression of NANOG in patients with colorectal adenocarcinoma. Cancer Biomark 2016;17:155-163.

25. Lomnytska MI, Becker S, Bodin I, et al. Differential expression of ANXA6, HSP27, PRDX2, NCF2, and TPM4 during uterine cervix carcinogenesis: diagnostic and prognostic value. Br J Cancer 2011;104:110-119.

26. Ostman A. PDGF receptors-mediators of autocrine tumor growth and regulators of tumor vasculature and stroma. Cytokine Growth Factor Rev 2004;15:275-286.

27. Jedroszka D, Orzechowska M , Bednarek AK. Predictive values of Notch signalling in renal carcinoma. Arch Med Sci 2017;13:1249-1254.

28. Wang $\mathrm{H}$, Yin $\mathrm{Y}, \mathrm{Li} \mathrm{W}$, et al. Over-expression of PDGFR-beta promotes PDGF-induced proliferation, migration, and angiogenesis of EPCs through PI3K/Akt signaling pathway. PLoS One 2012;7:e30503.

29. Li L, Xu M, Li X, et al. Platelet-derived growth factor-B (PDGF-B) induced by hypoxia promotes the survival of pulmonary arterial endothelial cells through the PI3K/Akt/Stat3 pathway. Cell Physiol Biochem 2015;35:441-451.

30. Chen $\mathrm{G}$, Li X, Yang $\mathrm{J}$, et al. Prognostic significance of cyclooxygenase-2 expression in patients with hepatocellular carcinoma: a meta-analysis. Arch Med Sci 2016;12:1110-1117. 
31. Feng W, Li L, Xu X, Jiao Y, Du W. Up-regulation of the long non-coding RNA RMRP contributes to glioma progression and promotes glioma cell proliferation and invasion. Arch Med Sci 2017;13:1315-1321. 


\section{Figure Legends}

Figure 1 PDGFRB expression is up-regulated in resistant MGC and PDGFRB promotes cell invasion, migration and proliferation. (A) Analysis of PDGFRB expressions through qRT-PCR in response or resistant MGC cells. (B) Immunohistochemical staining for PDGFRB in the specimens from response or resistant MGC patients. (C-D) Invasion and migration assays in response, restraint and PDGFRB shRNA resistant MGC cells. (E-F) MTT and colony formation assays showed that cell viability and proliferation ability in resistant group were stronger than that in response group and were inhibited by PDGFRB knock-down. ${ }^{*} P<0.05$ and $* * P<0.01$, compared with response group. ${ }^{\#} P<0.05$, compared with resistant group.

Figure 2 PDGFRB overexpression activates PI3K/AKT pathway and PDGFRB can be inhibited by treating with sunitinib malate in resistant MGC cells. (A) Network figure of MGC genes. The PPI data of MGC was gained from STRING. It indicated that PDGFRB involved in the occurrence and development of MGC. (B) The expressions of VEGFR, PTEN, PDGFRB were detected by Western blot in three groups (cells in response group and resistance group were transfected with empty vectors, resistant cells in shRNA group transfected with plasmid that PDGFRB gene was knocking down), all the expression levels were compared with $\beta$-actin expression. Results are indicated as mean $\pm \mathrm{SD}(\mathrm{n}=3) .{ }^{*} P<0.05$, compared with response group. ${ }^{\#} P<0.05$, compared with resistant group. (C) PDGERB expression level in resistant cells treated with three different inhibitors: sunitinib malate, crenolanib and sorafenib tosylate, comparing with 
vehicle were measured by qRT-PCR. ${ }^{*} P<0.05$, compared with vehicle group. (D) PDGERB expression level in resistant cells with various treatments were measured by qRT-PCR. ${ }^{*} P<0.05$, compared with vehicle group. ${ }^{\#} P<0.05$, compared with DCS group.

Figure 3 Inhibition of PDGFRB suppress the invasion and migration of cancer cells. (A) Transwell invasion assay showed that the invasion level of DCS + sunitinib malate group was dicreased compared with vehicle group as well DCS group. (B) Wound healing assay showed the migration ability in DCS + sunitinib malate group was lowest among three groups. (C) MTT assay for measuring the cell viability of cancer cells in three groups. (D) Colony forming assay showed that the colony numbers in DCS + sunitinib malate group was the lowest compared with other two groups. ${ }^{*} P<0.05$, compared with vehicle group. ${ }^{\#} P<0.05$, compared with DCS group.

Figure 4 Animal experiments for validating the effect of PDGFRB in metastatic gastric cancer. (A) Tumor volume in DCS + sunitinib malate group was smallest among three groups after 21-day observation. (B) The tumor weight in DCS + sunitinib malate group was also the lowest among three groups. (C) Immunohistochemical staining for PDGFRB revealed the protein expression in three groups. (D) qRT-PCR was performed to detect PDGFRB in excised tumor tissues. (E) and (F) Survival rate of MGC patients that treated with or without chemotherapy was assessed. 
Table 1 Differentially expressed genes between metastasis gastric cancer tissues and normal gastric tissues.

\begin{tabular}{|c|c|c|c|c|}
\hline Gene symbol & P. Value & $\mathbf{t}$ & B & $\operatorname{LogFC}$ \\
\hline $\mathrm{A} 1 \mathrm{CF}$ & 0.000359 & -3.9 & -4.38 & -2.11 \\
\hline MLN & 0.000606 & -3.72 & -4.39 & -2.56 \\
\hline ALDOB & 0.000749 & -3.65 & -4.4 & -2.65 \\
\hline FABP2 & 0.00103 & -3.54 & -4.41 & -2.45 \\
\hline ERICH4 & 0.001031 & -3.54 & -4.41 & -2.62 \\
\hline CXCL8 & 0.001378 & 3.44 & -4.42 & 2.41 \\
\hline SLC28A2 & 0.001988 & -3.31 & -4.43 & -2.42 \\
\hline CLCA1 & 0.002327 & -3.25 & -4.43 & -2.97 \\
\hline CHST5 & 0.002575 & -3.21 & -4.44 & -2.38 \\
\hline S100A8 & 0.004543 & 3.01 & -4.46 & 2.3 \\
\hline OTC & 0.00512 & -2.96 & -4.46 & -2.51 \\
\hline KLK10 & 0.005151 & 2.96 & -4.46 & 3.13 \\
\hline GKN1 & 0.005696 & -2.92 & -4.46 & -4.41 \\
\hline ZG16 & 0.006172 & -2.89 & -4.47 & -2.23 \\
\hline PDGFB & 0.007054 & 2.84 & -4.47 & 2.1 \\
\hline SI & 0.007502 & -2.82 & -4.47 & -2.72 \\
\hline CHP2 & 0.007885 & -2.8 & -4.47 & -2.86 \\
\hline TRIB3 & 0.008729 & 2.76 & -4.48 & 2.4 \\
\hline ALDOB & 0.008874 & -2.75 & -4.48 & -2.75 \\
\hline FCGBP & 0.00909 & -2.74 & -4.48 & -2.45 \\
\hline KRT20 & 0.00926 & -2.73 & -4.48 & -2.57 \\
\hline
\end{tabular}

FC, fold change referred to the change in metastasis gastric cancer tissues relative to normal gastric tissues. 
Table 2 Correlation between PDGFRB expression and clinic-pathological features.

\begin{tabular}{|c|c|c|c|c|}
\hline Variables & $\operatorname{High}(n=71)$ & Low $(n=31)$ & $X^{2}$ & $\boldsymbol{P}$ \\
\hline \multicolumn{5}{|l|}{$\operatorname{Age}(y)$} \\
\hline$\geq 50$ & $41(57.75)$ & $17(54.84)$ & 0.074 & $>0.05$ \\
\hline$<50$ & $30(42.25)$ & $14(45.16)$ & & \\
\hline \multicolumn{5}{|l|}{ TNM } \\
\hline Stage I II & $21(29.58)$ & $22(70.97)$ & 15.16 & $<0.05$ \\
\hline Stage III $\sim$ IV & $50(70.42)$ & $9(29.03)$ & & \\
\hline \multicolumn{5}{|c|}{ Differentiation degree } \\
\hline High degree & $12(16.90)$ & $24(77.42)$ & 34.802 & $<0.05$ \\
\hline Middle degree & $18(25.35)$ & $3(9.68)$ & & \\
\hline Low degree & $41(57.75)$ & $4(12.90)$ & & \\
\hline \multicolumn{5}{|l|}{ Tumor size $(\mathrm{cm})$} \\
\hline$<5$ & $34(47.89)$ & $17(54.84)$ & 0.417 & $>0.05$ \\
\hline$\geq 5$ & $37(52.11)$ & $14(45.16)$ & & \\
\hline
\end{tabular}


Table 3 COX regression analysis of the prognostic factors for MGC patients

\begin{tabular}{cccc}
\hline Variables & RR & $95 \%$ CI & $P$ \\
\hline TNM staging & 75.411 & $1.428 \sim 3.105$ & 0.013 \\
PDGFRB expression & 101.425 & $2.428 \sim 10.121$ & 0.002 \\
Differentiation degree & 81.401 & $2.716 \sim 4.379$ & 0.007 \\
\hline
\end{tabular}

Note: RR, relative risk; CI, confidence interval; MGC, metastatic gastric cancer. 


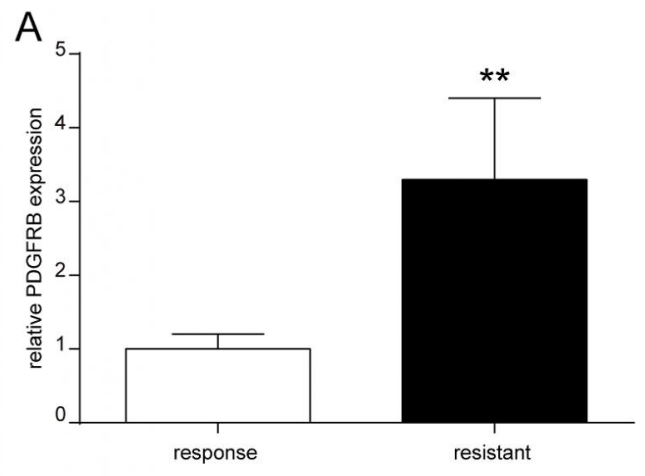

C


E

- - response

- resistant

^ shRNA

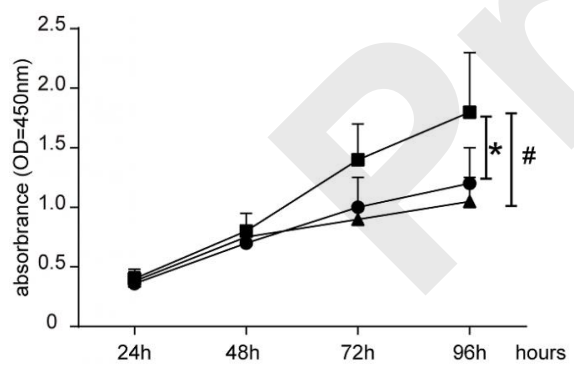

B

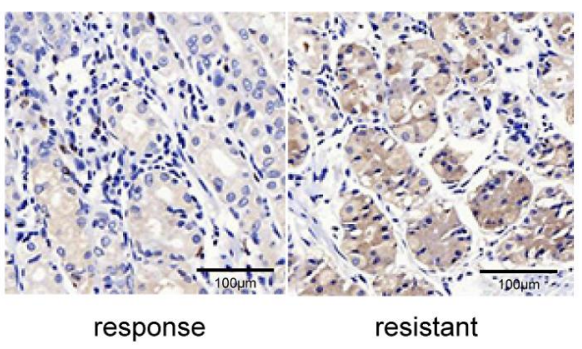

D
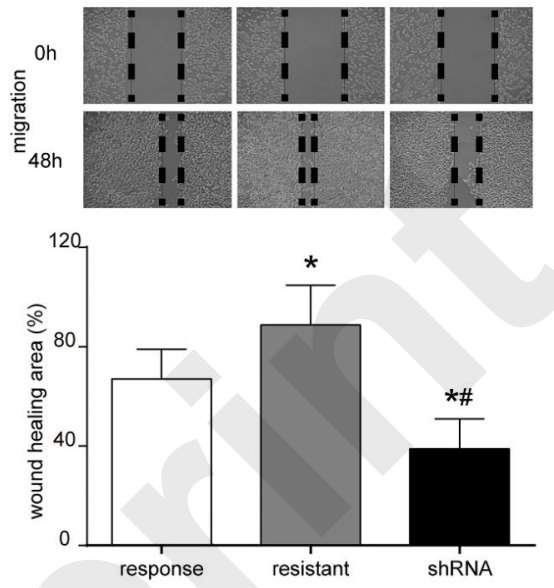

F
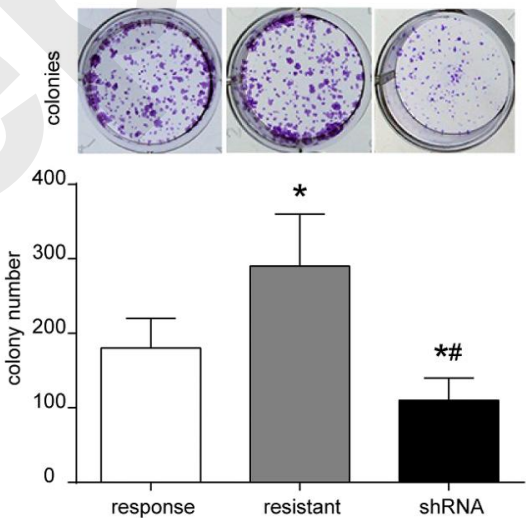

Figure 1 
A



C

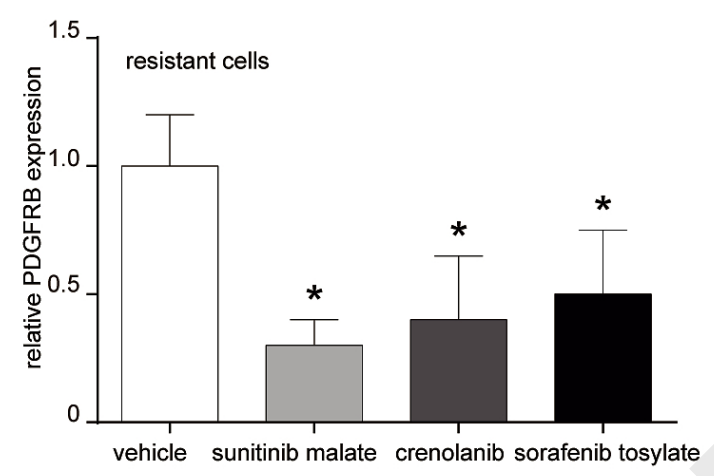



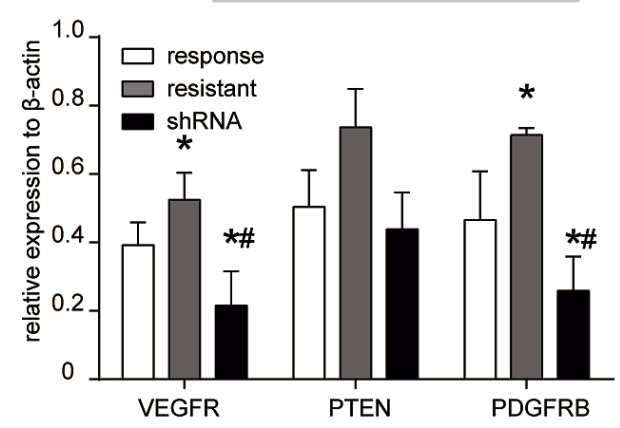

D



Figure 2 
A
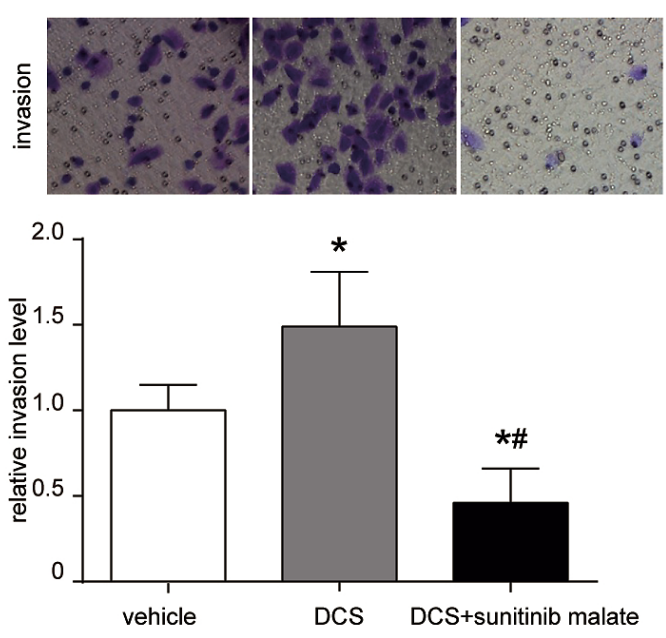

C



B
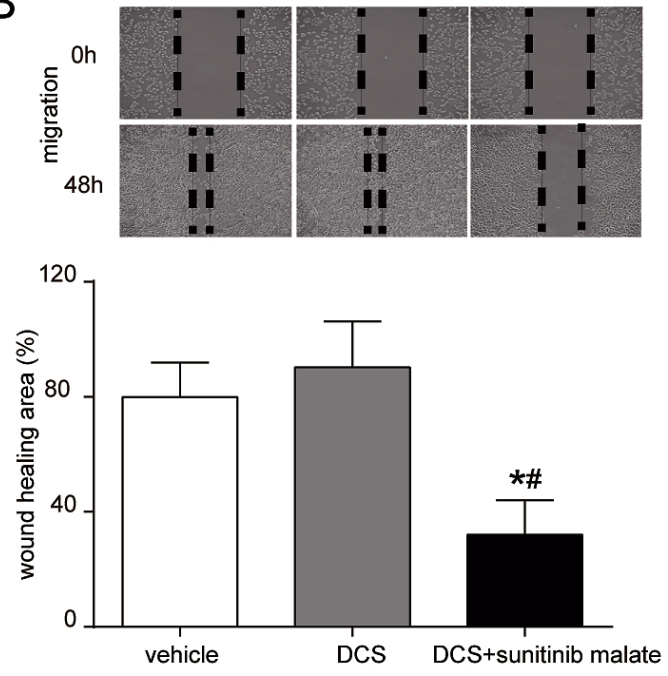

D
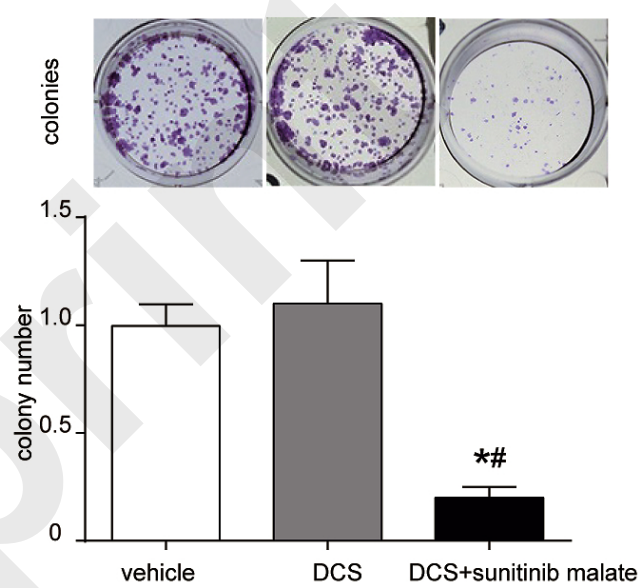

Figure 3 
A
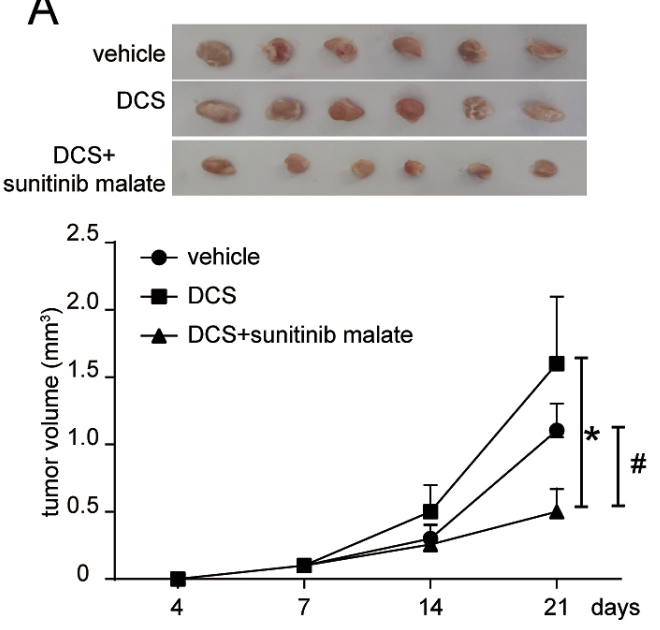

C

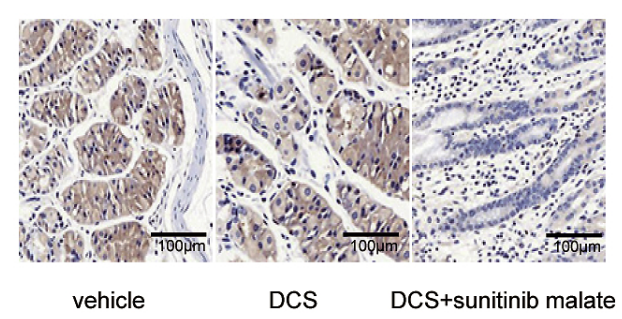

E



B

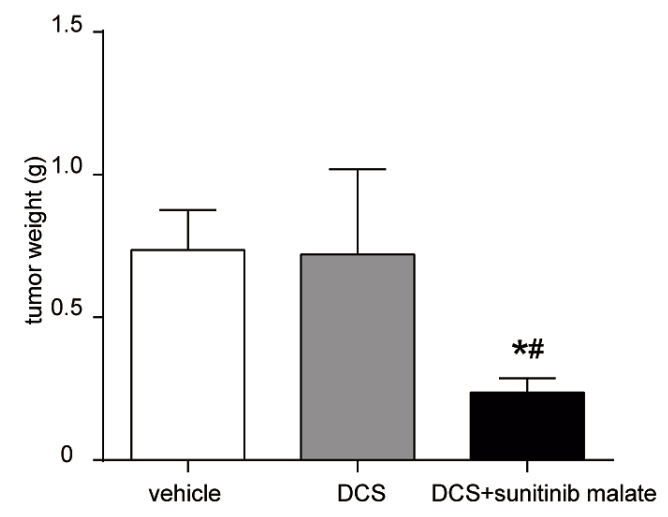

D



F

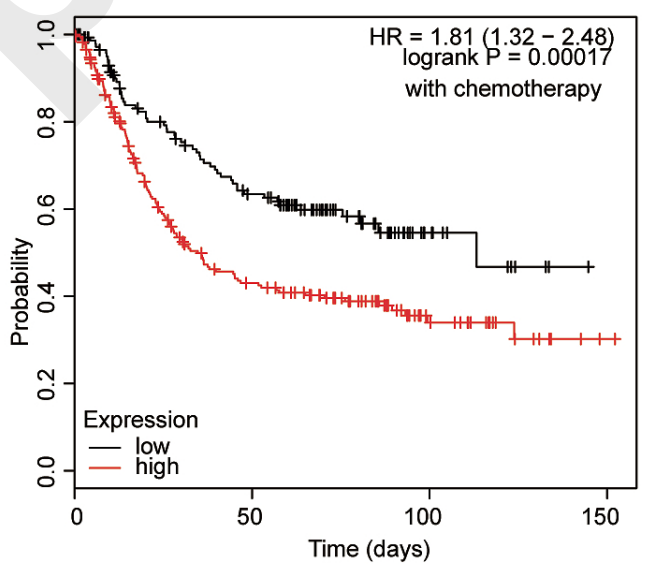

Figure 4 